\title{
Study of Gully Pot Monitoring System for Urban Areas
}

\author{
Deepthi.V, Karthika.K, Kowsika.R*, Siva Keerthana and R. Sudarmani
}

Department of Electronics and Communication Engineering, Faculty of Engineering, Avinashilingam Institute for Home Science and Higher Education for Women, Coimbatore - 641108 Tamilnadu, India

Received 16 Dec 2017, Accepted 17 Feb 2018, Available online 22 Feb 2018, Vol.8, No.1 (Jan/Feb 2018)

\begin{abstract}
Sewer failure occurs is one of the major problem in urban areas. The major cause of flooding is due to overflow of sewers. Several manholes across residential areas are overloaded due to excess sewage load. This is the main reason for the occurrence of Chennai flood. Inefficient gully pot network is the major reason for the over flooding of mainstream sewage pipes. There is a need for an efficient system design for monitoring a gully pot which is capable of providing required information about sewage block is prior to the occurrence. Wireless Sensor Network (WSN) has been used to monitor the sewer blockages. The blockage will be detected and the detected information has been transmitted to the authority through a GSM and developed in mobile application.
\end{abstract}

Keywords: Gully pot, Sensors, WSN, GSM, Mobile application.

\section{Introduction}

Gully pots can be defined as the centralized collection or storage point for the sewage coming from nearby household from which it goes into the mainstream of sewage pipes and drained into the processing plants for further treatment. An efficient design is required for Gully pot monitoring system which is capable of providing sufficient information about the sewage water level and ph of the sewage and also to identify the presence of toxic gases produced in the gully pot to avoid human causalities.

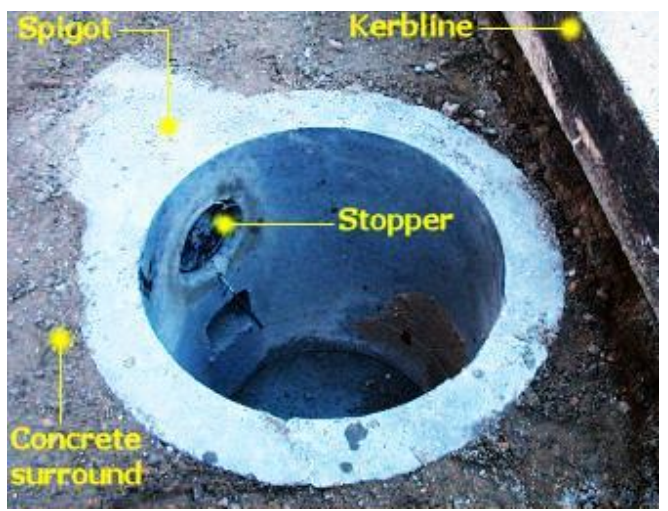

Fig 1: Concrete gully pot

Wireless sensor network is used to gather information regarding real time events that has to be processed, stored and saved for future use.

*Corresponding author's ORCID ID: 0000-0002-8971-8435, DOI: https://doi.org/10.14741/ijcet/v.8.1.25

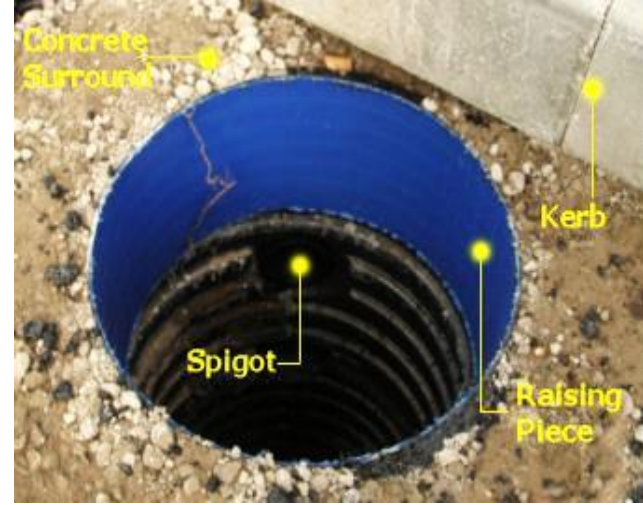

Fig 2: Plastic gully pot

The gathered information is transferred to main sewer using sensor node.WSN are used for intelligence monitoring of temperature, humidity, water level, presence, vehicular activity, remote health monitoring of several patients. The rural and suburban environment causes less distortion in wireless communication networks than in the urban areas. Wireless sensor network are being deployed in sewers for sewage flood monitoring and also to monitor methane and hazardous gases. This is used as a solution for disaster relief strategy.

In earlier days gully pot monitoring is done using CCTV.CCTV is expensive and data interpretation can be done only by manual method. This problem was overcome by using WSN technique. In this paper, literature review is explained in section II and end with conclusion. 


\section{Literature survey}

1) A low power WSN for gully pot monitoring in urban catchments

Chan H.See et al (2012), proposed the concept, where WSN is used for monitoring the gully pot. The technique involved in monitoring the process includes zigbee sensor node, GPRS and wireless mesh networt. Zigbee consists of transceiver and acoustic sensor probe, to sense the level of water flow. GPRS is used to track the location of the block. Low power adaptive mesh network topology has been implemented to increase the radio coverage and communication link. Data gatherer gathers the information from sensor nodes and displayed at webpage. Drawback of this paper is that zigbee is used for short distance communication and sensor reliability is also affected.

\section{2) Energy efficient gully pot monitoring using RFID}

R.A.Abd-Alhameed et al (2013), proposed the concept of RFID technique to monitor the gully pot. An RFID system comprises of RFID tag, reader, software and database. RFID tags are used communication through radio waves. Hence the object can be identified and tracked.The reader scans the tags and transmit the information to the database .This technique is not implemented in real time application but has an experimental setup. The coverage area is not specified.

3) An integrated wireless sensor network for monitoring gully pot drainage, landslides and waste gas measurement

G.Kuppulakshmi et al (2013), described the concept of the Integrated WSN system which deals about the catastrophic warning on potential blockage to prevent the sewer failure in drainage system, flow occurrence during landslides, waste gas development, monitor system using wireless sensor. The main issue with blockage formation is its intermittent nature so that current inspection technology used in this system was not be efficient to detect the serious sudden incidents and serious blockages. The disaster which is found as catastrophic such as landslides cannot be predicted before it occurs. Formation of waste gas cannot be predicted. This is an Advanced Metering Infrastructure (AMI) associated sensor network which consists of smart meter, Accelerometer sensor, ultrasonic sensor, gas sensor. Gas drainage flowmeter is attached to detect the gas drainage borehole block with time.

4) An integrated sensor network to enhance the performance of gully pot monitoring

Karthik saran M et al (2017), discussed the concept of better processing of sewage and to avoid flooding risk during heavy rain in metropolitan areas. To tackle this problem ultrasonic sensor is used. According to the blockage size, the propagation delay had been changed.
It is used to detect the object within set area output will change state which is visualized by integrated LED Gas sensor to sense the level of gas when specific amount of gas is introduced. $\mathrm{Ph}$ sensor uses $\mathrm{Ph}$ meter to measure the concentration of hydrogen ions and finally Level sensor to detect the level of sewage that is present in Gully pot which can be used to give the information to the station about sewer water at particular time.

\section{5) Sewernort: A drifting sensor for in still waste water collection system gas monitoring}

Lim JS et al (2011), proposed the floating sensor for sewage identification. The floating sensor measures concentration of gas as well as location of the sewer coordinates. The main objective for monitoring the sewer gas is to indicate sewer condition. If the gas level is low, then the sediment deposit on the ground is also low. The major drawback of this system is that,the sensor had to be retrieved to gather the data and then it has to be transmitted to the authorities.

6) Automation of smart waste management using IoT to support Swachh Bharat Abhiyan-practical approach

Bharadwaj B et al (2017), developed the concept to collect the dry and wet waste separately, which is to be placed in conveyor belt. This system consist of the following technique: 1)RFID 2)IoT. RF technology has been used to communicate between microcontrollers and switching buttons, LCD is used to display the status of techbin. IoT has been used as a mobile communication network. The signals are sending to a web based software application which can be viewed by the management.

7) Methane and carbon monoxide gas deduction system based on semiconductor sensor

Emilcordos et al (2006), used a single sensing element to detect $\mathrm{CO}$ and methane. The sensor used to detect the CO and methane gas is SB95.It is a semiconductor sensor. It detects the concentration of the gases and modifies the resistance accordingly. It is also possible to detect the fluctuation of the signal when it passed into microcontroller, where the analog signal gets converted into digital signal. The alarm light indicated as red will denote the excess limit of methane whereas excess of $\mathrm{CO}$ is denoted in yellow colour. Alarm also accompanied with the buzzer.

\section{8) IoT based smart waste management system}

AkshayBhalerao et al (2017), proposed the concept where the ultrasonic sensors are used to sense the water level if the level of sewage rises. It records the threshold value and sends the notification to the sewer using Wi-Fi module. GPS module is used to send the coordinates of the manhole. Sewage cleaners were 
notified about the block through android app and they rectified the block in the sewage .For better wireless connectivity and GPS monitoring lids had small hole covered with IP67 material. Threshold level varies according to the variation in their heights.

\section{9) New Approach for wireless Underwater Identification: Acoustic frequency Identification (AFID)}

Nasri Nejah et al (2011), proposed the concept of Underwater Identification techniques raise several potential applications and issues. In this paper, wireless identification system for underwater application was proposed. International Morse code (IMC) is a practical solution in detecting, locating and identifying underwater equipment. An acoustic wave is used as physical transmitter medium in underwater wireless communication. Acoustic frequency Identification (AFID) technique is based on identifying technique used in $\mathrm{RF}$ environment. Acoustic identification system can handle the constrain of underwater channel.

\section{0) RFID tag antenna based sensing: Does your beverage glass need a refill?}

Ragul Bhattacharyya et al (2010) proposed the concept where the liquid level detection in beverage glass is done with the help of RFID tag. RFID technology is a viable technology for track and trace applications. This technique is of low cost and standardised. This technique is used for sensing which is integrated with RFID chip. RFID tag antenna acts as sensor. This helps in detection whether the glass is empty or full, and also whether it is possible to localise an empty glass. This is a less intrusive sensing methodology for a problem like fluid volume detection in glass. The larger detection range associated with ultra-high frequency RFID allows to deploy reader antennas in unobtrusive locations.

\section{1) Online waste water monitoring system using solid state sensor}

Vincenzo Di Lecce et al(2010), implemented online waste water monitoring system used solid state sensor. This paper portraits proper water and wastewater management in urban cities. Municipal waste water is contaminated with chemicals like carbon - di - oxide, nitrogen - di -oxide, BOD5 etc. Industrial waste water contains chemicals like ammonia nitrate and carbon di - oxide, sulphur. Regular monitoring of this system provides clean and smart cities. Here, Vapour Phase E Nose is deployed in the pipeline or in the drainage system. The main objective of vpen is to monitor the water quality in real time. Depending upon the gas, various gas detection sensors has been inserted. The microprocessor has been used as processing unit. The sensors used here is 8TGS Figaro gas sensors, because it is sensitive to wide spectrum of gas. It is fixed in stainless steel sensor chamber. This system uses low cost solid sensor, but sensors performance was poor and required advanced technique.

\section{2) Dynamic water gate assignment scheme for data aggregation in long-thin sensor networks}

You-Chiun Wang et al (2010), discussed about WSN facilitates monitoring of physical environments. Technique sensors are deployed to form a network with regular topology. Long thin network topology has been used for WSN applications, which is used for monitoring the quality of water in the sewers. The Long thin(LT) topology consists of long branches and each branch consists of tens or hundreds of sensors. For each sensor, along the branch there is one potential parent node towards the sink. Branch nodes connect all the branches. By viewing the LT with $\mathrm{n}$ sensor as shortest path tree rooted at the sink. Each sensor transmits one packet to the sink without data aggregation. WSN requires 62 transmissions to collect all sensed data.

WADA is a dynamic water gate assignment scheme for data collection, which groups all the sensors. Here, hundred sensors had been deployed to collect the data The minimum distance between each sensor is $15 \mathrm{~cm}$. The microprocessor has been used to collect the required data from the sensor and zigbee protocol used for communication protocol. By using WADA scheme, many number of sensors can be aggregated and sensors overlapping had been avoided .WADA reduces amount of data transmission of sensors in an effective manner.

\section{3) A zigbee based wireless sensor network for sewerage monitoring}

C.H.Cee,S.J.Tait et al(2009), in this paper the concept of practical deployment of WSN in urban areas for drainage monitoring was analysed. The manual operations can be replaced by a telemetry analysis. But telemetry analysis is also cost effective. Hence, low cost WSN has been preferred due to its cost effective nature. WSN had been implemented using Zigbee communication and acoustic sensor technologies to monitor the water levels. Cross bow mica2 sensor nodes, which is tiny is used. Acoustic sensors are used to measure the water levels. The received signal level was transferred to the Data Acoustic Circuit board, in order to indicate the state of water level. The antennas are implemented in this system to overcome limited communication range of low power mesh network. Stargate platform had used as a data gatherer which is mounted on the lamppost. GPRS is used to transfer the received data from stargate to remote server. In this system sensors reliability is poor.

\section{4) A wireless mesh monitoring system for sewage treatment plant}

Litingcao (2009), used the wireless communication system rather than wired monitoring system in the 
gullies. It consists of four blocks: sensor nodes, data collector (gate way), monitoring system and wireless communication networks. Many sensor nodes are deployed in the field to gather the information regarding temperature, dissolved oxygen, $\mathrm{Ph}$ level and so on. The gathered information from sensor nodes had been transferred to data collector using Zigbee wireless mesh network. The data collector transmits data to monitoring system using Ethernet. Here, the data collector acts as a gateway monitoring system has been designed based on FPGA. Microchips PIC18LF4620 acts as a processor for designing Zigbee node. CC2520 wireless chip adapted as communication unit. Routing protocol based on genetic arithmetic is adapted for this system. Depending upon the areas the selection of sensors varies.

\section{5) Development of arm7 based sensor interface for industrial wireless sensor network in IoT Environment}

T.Balakrishna et al (2016), analysed the performance and various functional characteristics of ARM based WSN, for monitoring the parameters of $\mathrm{CO} 2$ concentration, relative humidity around the pipeline.ARM based WSN has the feature of continuous monitoring with easy insertion procedures and high battery life time. Zigbee module is used to transmit the data to the remote location. The gain amplifier is used to amplify the measured sensors output. The maximum gain of the amplifier is the reference voltage of the analog to Digital Convertor. The filtered analog signal is given to the microcontroller's ADC module. Here, filter is used to reduce the noise level in the sensor output. Co sensors are used to detect the LPG, i-butane, propane, methane, hydrogen. GPRS is used to track the location of the block in the pipeline and transfer the required data to the corresponding authorities. The collected data is stored in cloud and also the data can be viewed using buzzer and LCD display.

\section{Conclusion}

Sewage failure occurs in urban areas is one of the major problem. Several severe problems are caused due to wastes. The waste will overflow due to the blockage that occurs inside the gully pot. The research papers are dealt with different methodologies by using various technologies for effective removal of blockages in gully pot. The concepts discussed in this paper will help the researchers who are doing research in these areas.

\section{Reference}

Chan H.See, Kirill V.Horoshenkov, RaedA.Abd-Alhameed, Yim Fun Hu, SeniorMember,IEEE, and Simon J.Tait (May 2012)A low power WSN for gully pot monitoring in urban catchments, IEEE sensors journal,vol.12,no 5,
A.Atojoko, N. Mjaan, F.Elmgri, R.A.Abd-Alhameed, C.H.See,J.M.Noras (November 2013), Energy efficient gully pot monitoring using RFID, 2013 Loughborough Antennas \& propagation conference,, Loughborough, UK

G.Kuppulakshmi, J.Vaijayanthimal (May 2013),An integrated wireless sensor network for monitoring gully pot drainage , landslides and waste gas measurement, ISSN 2229-5518 International Journal of scientific and Engineering Research, Volume 4,Issue 5,

Karthik saran M, Aswin S M, Gobinath C (March 2017), An integrated sensor network to enhance the performance of gully pot monitoring, IJARMATE Vol.3, special issue 13

Lim JS, Kim J, Fried man J, LieeU,Vieiara L, Rosso D, et al (2011) Sewernort: A drifting sensor for in still waste water collection system gas monitoring. Ad Hoc Netw11:1456-71.

Bharadwaj B, M K umudha, Gowrichndra N, Chaithra G (February 2017), Automation of smart waste management using IoT to support Swachh Bharat Abhiyan- practical approach, Published in Computing and Communications Technologies (ICCCT), 2017 2nd International Conference on IEEE.

Emilcordos, ludovicferenczi, Sergiucadar, Simonacostiug, Gabriela PITL, Adrian ghita (May 2006): Methane and carbon monoxide gas deduction system based on semiconductor sensor in Automation, Quality and Testing, Robotics, 2006 IEEE International Conference on.

Akshay Bhalerao, Anima Ghosh, Siddhita Mhatre, Sayalee Vadgaonkar, Pallavi Wajge \& Ninad Shinde IoTbased smart waste management system,Published in IJCTA,10 (8), ISSN:0974-5572.

Nasri Nejah, Kachouri Abdennaaceur Andriux Laurent \& Samet Mounir (March 2011)New Approach for wireless Underwater Identification: Acoustic frequency Identification (AFID), published in Communications, Computing and Control Applications (CCCA), 2011 International Conference on IEEE.

Ragul Bhattacharyya, Christian Floerkemeier and Sanjay Sharama (2010) RFID tag antenna based sensing : does your beverage glass need a refill ?, Published in RFID, 20 May 2010 IEEE International Conference

Vincenzo Di Lecce, DomenicoPetruzzelli et al (July2016)Online waste water monitoring system using solid state sensors, Published in IEEE Environmental, Energy, and Structural Monitoring Systems (EESMS), 2016 IEEE Workshop

You-Chiun Wang, Che-Hsi Chuang et al (December 2010)Dynamic water gate assignment scheme for data aggregation in long-thin sensor networks, Published in IEEE Computer Symposium (ICS), 2010 International.

C.H.Cee,S.J.Tait et al (September 2009)A zigbee based wireless sensor network for sewerage monitoring,Published in IEEE Microwave Conference, 2009. APMC 2009. Asia Pacific

Litingcao (2009) A wireless mesh monitoring sysytem for sewage treatment plant published in IEEE Computing, Communication, Control, and Management, 29 September 2009. CCCM 2009.ISECS International Colloquium.

T.Balakrishna,R.Nagasweetha (May 2016), Development of arm7 based sensor interface for industrial wireless sensor network in IoT Environment,published in International Journal of Eminent Engineering Technologies, Volume 4, Issue $3^{\text {rd }}$ 